\title{
Pore-scale simulation of water/oil displacement in a water-wet channel
}

\author{
Jin Zhao ${ }^{1}$, Guice Yao ${ }^{1}$, Dongsheng Wen $(\bowtie)^{1,2}$ \\ 1 School of Chemical and Process Engineering, University of Leeds, Leeds LS2 9JT, UK \\ 2 School of Aeronautic Science and Engineering, Beihang University, Beijing 100191, China
}

(C) The Author(s) 2019. This article is published with open access at link.springer.com and journal.hep.com.cn

\begin{abstract}
Water/oil flow characteristics in a water-wet capillary were simulated at the pore scale to increase our understanding on immiscible flow and enhanced oil recovery. Volume of fluid method was used to capture the interface between oil and water and a pore-throat connecting structure was established to investigate the effects of viscosity, interfacial tension (IFT) and capillary number $(\mathrm{Ca})$. The results show that during a water displacement process, an initial continuous oil phase can be snapped off in the water-wet pore due to the capillary effect. By altering the viscosity of the displacing fluid and the IFT between the wetting and non-wetting phases, the snapped-off phenomenon can be eliminated or reduced during the displacement. A stable displacement can be obtained under high $\mathrm{Ca}$ number conditions. Different displacement effects can be obtained at the same $\mathrm{Ca}$ number due to its significant influence on the flow state, i.e., snapped-off flow, transient flow and stable flow, and ultralow IFT alone would not ensure a very high recovery rate due to the fingering flow occurrence. A flow chart relating flow states and the corresponding oil recovery factor is established.
\end{abstract}

Keywords VOF, pore scale, immiscible displacement, EOR, snap-off, $\mathrm{Ca}$

\section{Introduction}

Immiscible flow in porous media is fundamental to a range of applications such as underground pollutants transport, oil spillage and treatment, soil remediation and enhanced oil recovery (EOR), where pore scale understanding of the phase displacement is crucial. Pore scale ranges from a few micrometres to millimetres where the capillary force dominates the displacement process [1-3]. Previous

Received September 14, 2018; accepted March 1, 2019

E-mail: d.wen@buaa.edu.cn pore-scale study of multiphase flow has been focused on EOR, and the fluid displacement process is investigated experimentally by X-ray computed micro-tomography techniques for different core samples [4-6]. Pore-scale modelling by computational fluid dynamics (CFD) method has recently been established as a complimentary to the costly core analysis method. It is being used in the petroleum and environment sectors to model multiphase flow in column/core samples and to predict the effects of various dependent factors such as rock type, interfacial tension (IFT) and wettability [7].

There are generally two main methods to model the phase displacement. Interface tracking method based on lagrangian algorithms is normally not well suited for flow with interfaces undergoing complex deformations (such as coalescence or break up). In contrast, interface capturing method based on Eulerian algorithms is more appropriate for complex interface motion [8,9]. Volume of fluid (VOF) method was first introduced by Hirt and Nichols [10], which treated the immiscible phase as a single fluid with variable properties. Many studies have shown that VOF method is able to model multiphase flow through porous media with sub-pore resolution and to capture detailed interfacial phenomena, such as viscous deformation of the meniscus, snap-off and coalescence, jumps and abrupt reconfiguration of the interface [11-17].

At the pore scale, capillary force plays an important role in the fluid displacement. For example, in the EOR applications, a dominant mechanism for residual oil left in reservoirs is the "snap-off" phenomenon, which has been extensively studied. For oil droplets in water-wet pores, Roof [18] first proposed a structural criterion to determine the snap-off occurrence in circular pores. According to this classical model, snap-off occurs when $\frac{1}{R_{\mathrm{b}}}<\frac{1}{R_{\mathrm{t}}}+\frac{1}{R_{\mathrm{c}}}$, where $R_{\mathrm{b}}$ and $R_{\mathrm{t}}$ are the effective radii of the pore body and pore throat respectively, and $R_{\mathrm{c}}$ is the transverse radius of the throat curvature. The dependence of the snap-off on local geometry has been experimentally confirmed by Kiss 
et al. [19] and $\mathrm{Yu}$ and Wardlaw [20]. Extending from Roof's criterion for circular pores, Ransohoff et al. [21,22] proposed a quasi-static criterion for non-circular pores by using a dimensionless interfacial curvature, which varied for capillaries with different cross-sectional shapes. Later, Al-Gharbi et al. $[23,24]$ presented a dynamic pore-scale network model that could predict the meniscus oscillations and snap-off phenomena observed in micromodel experiments. Beresnev and Deng [25] proposed a nonlinear dimensionless equation as a purely geometric criterion to describe the dynamics of snap-off and calculated the snapoff time, which was verified by the results of CFD simulations [26,27]. Deng et al. [28,29] further extended Roof and Ransohoff criteria by considering the imbalance between the pore body and the pore throat, considering a wetting film existing between a non-wetting fluid and wall. Roman and Abu-Al-Sand et al. [30,31] developed a multiscale sharp-interface level-set method to investigate the snap-off coalescence phenomenon for immiscible twophase flow with a pre-existing thin film on solid surfaces, whose results were validated by comparison with both theory and experiments. Recently, Deng et al. [32] analysed the dynamics of the wetting/non-wetting interface instability in sinusoidal constricted capillary tubes by using a theoretical model to couple the wetting film thickness to the local capillary number $(\mathrm{Ca})$ at the pore throat. Although the pore-scale snap-off process has been extensively investigated, there are still three main limitations: (i) most of previous research was based on core-scale study with a main focus on the flow displacement in a crossing pore network, and detailed study at a single pore scale is still insufficient; (ii) previous pore-scale studies are mainly focused on super-critical carbon dioxide as the non-wetting fluids, which cannot represent water/oil displacement suitable for EOR and oil remediation applications; (iii) no study has been investigated on potential ways to suppress the snap-off process at the pore scale to decrease the amount of trapped oil.

On the other hand, to suppress the snap-off phenomenon at the pore scale, many chemicals have been used after the secondary water flooding process, which is termed as chemical EOR [33]. The main idea is to increase the value of the $C a, C a=v \cdot \mu / \sigma$, (i.e., decreasing the IFT by using surfactants, or increasing the invading fluid's viscosity by using polymers), and to decrease the snap-off probability, leading to a reduction of the residual oil saturation. Though many core flooding experiments have been performed [3437], only very limited studies have been conducted at the pore scale, with inconsistent results reported [38-43]. Ultralow IFT injection has also been proposed recently as a promising EOR technology; however, it is still debatable on the exact role that IFT plays in the process [33]. It has been accepted generally that a high $\mathrm{Ca}$ can lead to a large oil recovery factor, regardless the modification of viscosity or IFT.

Using EOR as an example, this work performs a fundamental flow study at the single pore scale to increase our understanding of oil displacement and water/oil flow characteristics based on the VOF method. A pore-throat connecting channel is established under water-wet condition and a parametric study of the effect of viscosity and IFT at the same $C a$ on oil displacement is performed, leading to the establishment of a flow map and the identification of the different roles of viscosity and IFT.

\section{Mathematical methods}

The two phase flow of oil and water in this simulation is described by the incompressible Navier-Stokes equations:

$$
\left\{\begin{array}{c}
\frac{\partial}{\partial t} \rho \boldsymbol{u}+\rho \nabla \cdot(\boldsymbol{u} \boldsymbol{u})=-\nabla p+\mu \nabla \cdot\left(\nabla \boldsymbol{u}+\nabla \boldsymbol{u}^{\mathrm{T}}\right)+\boldsymbol{F}+\rho \boldsymbol{g} \\
\nabla \cdot \boldsymbol{u}=0
\end{array}\right.
$$

where $\boldsymbol{u}$ is the velocity vector, $\rho$ is the density fluid, $\mu$ is the viscosity of fluid, $p$ is the static pressure, $\boldsymbol{F}$ is the general body force which is the volume surface tension $\boldsymbol{F}_{\mathrm{vol}}$ in this simulation, and $\boldsymbol{g}$ is the gravitational acceleration, which is neglected in this simulation due to its minor influence at the pore scale. The superscript " $T$ " denotes the matrix transpose.

The finite-volume based the CFD code FLUENT is used to solve the Navier-Stokes equations numerically. The VOF method is adopted to describe oil/water two phase flow. The volume fraction equation is used for tracking the interface between multiple phases:

$$
\frac{\partial\left(\alpha_{i} \rho_{i}\right)}{\partial t}+\nabla \cdot\left(\alpha_{i} \rho_{i} \boldsymbol{u}\right)=\rho_{i} S_{\alpha_{i}}+\sum_{j=1}^{n}\left(\dot{m}_{j i}-\dot{m}_{i j}\right),
$$

where $m_{j i}$ and $m_{j i}$ are the mass transfer from each other between phase $i$ and phase $j$, both $m_{j i}$ and $m_{j i}$ are zero in this simulation due to the no mass-transfer assumption between water and oil phase in this work, $S_{\alpha_{i}}$ is the source term of $i^{\text {th }}$ fluid, $\rho_{i}$ is the density of $i^{\text {th }}$ fluid, and $\boldsymbol{u}$ is the flow velocity vector. The volume fraction $\alpha_{i}$ is defined as follows:

$$
\left\{\begin{array}{l}
\alpha_{i}=0: \text { the cell is empty with no traced fluid inside } \\
0<\alpha_{i}<1: \text { the cell contains the interface } \\
\alpha_{i}=1: \text { the cell is full of the } i^{\text {th }} \text { fluid } \\
\sum_{p=1}^{n} \alpha_{p}=1: \text { the cell must be filled with either single fluid phase or a combination }
\end{array} .\right.
$$


The fluid properties at the interface are given by $\left\{\begin{array}{l}\rho=\sum \alpha_{i} \rho_{i} \\ \mu=\sum \alpha_{i} \mu_{i}\end{array}\right.$, which are averaged volume fraction for an $n$-phase system.

The continuum surface force (CSF) model [44] is used to calculate the curvature of the interface and volume surface tension. In the CSF model, the volume surface force has the following form: $\boldsymbol{F}_{\mathrm{vol}}=\sigma \kappa \nabla \alpha_{i}$ where $\sigma$ is the IFT, and $\kappa$ is the curvature, which is defined as: $\kappa=\nabla \cdot \boldsymbol{n}$, where $\boldsymbol{n}$ is the unit normal vector of the interface, $\boldsymbol{n}=\frac{\nabla \alpha_{i}}{\left|\nabla \alpha_{i}\right|}$. The pressure based unsteady solver is used for the simulation by using the following algorithm: the pressure-implicit with splitting of operators for pressurevelocity coupling, the least squares cell-based scheme for gradient spatial discretization, the pressure-staggered option for pressure spatial discretization, the geometric reconstruction approach for volume faction spatial discretization, and the first order explicit scheme for temporal discretization.

\section{Physical model}

\subsection{Pore geometry}

According to the previous studies $[45,46]$, pore-throat sizes (diameters) are generally greater than $2 \mu \mathrm{m}$ in conventional reservoir rocks. Geometrical reasoning and experimental data suggest that conventional reservoir rocks have aspect ratios, i.e., the ratio of pore body size to pore throat size, in the range of $5: 1$ to $10: 1$ [47]. In this work, a twodimensional pore body-throat connecting channel is proposed with an aspect ratio of 7:1 as the standard case, shown in Fig. 1. The radius of the connecting pore throats $d / 2$ is $2.5 \mu \mathrm{m}$, and the radius $R_{1}$ of the circular pore is $17.5 \mu \mathrm{m}$. To smooth the sharp corners in the geometry, the connectivity between pore bodies and throats are optimised by a curve with the radius of $R_{2}=2.5 \mu \mathrm{m}$. The side length $L$ of the pore-throat connecting channel is $175 \mu \mathrm{m}$. A unit length in the $z$ direct perpendicular to the figure is applied to allow 2D simulation. This represents a typical geometry in microchips used for pore-scale study and some reservoir pores. The shadow region refers to the solid reservoir rock. Due to the scale of the channel, the gravity effect is neglected in this work. For such simple geometries, it has been shown by many studies that $2 \mathrm{D}$ simulation is adequate to capture the complex multiphase flow dynamics $[2,8,25,28,32]$, and is adopted in this work.

\subsection{Fluid properties}

The properties of crude oil and water are affected by various factors, such as temperature and pressure. Considering that during a flooding process, the reservoir

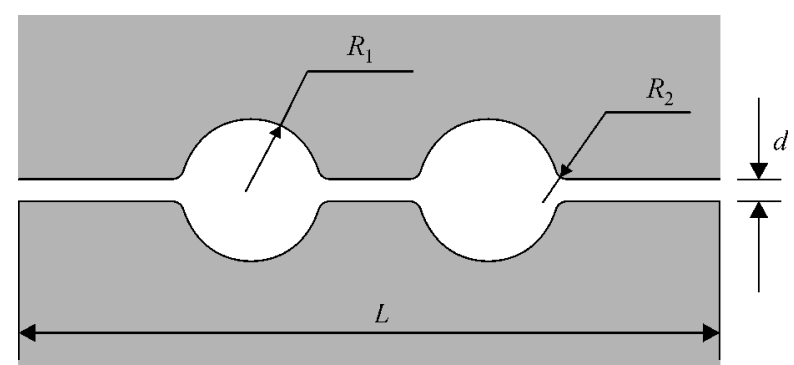

Fig. 1 Schematic view of the two-dimensional pore-throat configuration.

pressure and temperature are generally constant, constant fluid properties are assumed, and no heat transfer occurs between the two-phase flows. A pure water-wet sandstone pore surface is assumed here. According to the typical oilrecovery applications, assuming the water viscosity of $0.001 \mathrm{~Pa} \cdot \mathrm{s}$ and a typical oil viscosity of $0.01 \mathrm{~Pa} \cdot \mathrm{s}[48,49]$, the ratio of $\mu_{\mathrm{w}} / \mu_{\mathrm{o}}$ for the standard case is set as 0.1 . Both the density of the core fluid and displacing fluid are set as $1000 \mathrm{~kg} \cdot \mathrm{m}^{-3}$ [27]. The standard oil/water IFT with a value of $0.05 \mathrm{~N} \cdot \mathrm{m}^{-1}$ is taken from the reference [11].

\subsection{Initial and boundary conditions}

The initial interface configuration used in this work is shown in Fig. 2, which initial oil saturation is $73.97 \%$. The red part represents the oil phase, while the blue part represents the water phase. A uniform water-film thickness of $1.5 \mu \mathrm{m}$ was used as an initial pattern for a completely water-wet situation, where the contact angle is zero between the aqueous phase and the rock surface.

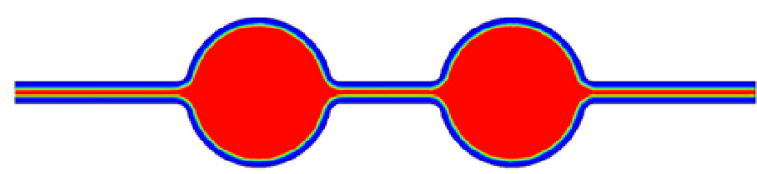

Fig. 2 The initial water-wet pore configuration.

The boundary condition for the left inlet is the velocity inlet, where the inlet velocity with $0.005 \mathrm{~m} \cdot \mathrm{s}^{-1}$ of the invading phase (water) is calculated from the average flow rate used in a typical EOR operation. It is obtained by assuming water as the reservoir's most abundant phase and Darcy's Law applied. It states that $Q_{\mathrm{D}}=\left(\frac{\kappa^{*} \cdot A}{\mu_{\mathrm{w}}}\right) p_{x}$, where $\kappa^{*}$ is the permeability, $A$ is the cross-sectional area, $\mu_{\mathrm{w}}$ is water viscosity and $p_{x}$ is the background pressure gradient. To convert $Q_{\mathrm{D}}$ to an estimated velocity in a single channel, below equation is obtained, $u=\left(\frac{\kappa^{*}}{\mu_{\mathrm{w}} \cdot \varphi}\right) p_{x}$, where $\varphi$ is the porosity. The maximum background pressure gradients of a typical oil field, $p_{x}$, are on the 
order of $10^{6} \mathrm{~Pa} / \mathrm{m}$, and the high end of reservoir permeability $\kappa^{*}$ is on the order of $10^{-11} \mathrm{~m}^{2}$ [50]. For most reservoir rocks, porosity varies from less than $1 \%$ to $40 \%$, thus the $C a$ varies from an order of $10^{-2}$ to $10^{-6}$. The outlet of the pore is specified as the pressure outlet. The boundary conditions for the pore wall are the adiabatic boundary and no-slip condition. The constant total flow rate is maintained in this work.

\section{Results and discussions}

\subsection{Grid independency and numerical validation}

Grid-refinement studies are performed to ensure that the computed profiles and the time evolution of the fluid-fluid interface are grid independent. The structured rectangular grid is used for simulations in this study with refined grids near the boundaries, generated by the meshing code ICEM CFD. Initially, a grid independence study is performed in a water-wet system originally saturated with oil. A time evolution of $10^{-9} \mathrm{~s}$ is used for calculations to satisfy the Courant number and Brackbill constrains.

The process of water flooding through an oil-saturated pore is applied as a classic and standard example of multiphase flow dynamics for the grid independency test here. Figure 3 shows the water/oil distributions after the flooding time of 10.0 and $100 \mathrm{~ms}$ in the displacement process at different mesh resolutions. The oil/water distributions are nearly the same, with some small variations at the water/oil interface. The higher the resolution, the sharper the interface is. Based on the grid sensitivity test results, the differences of the predicted pressure drop among the five grid systems are 9.74\%, $6.04 \%, 3.68 \%, 0.46 \%$, and $0.28 \%$ respectively. Therefore, considering both computational accuracy and efficiency, the fourth mesh system with 35276 computational grids is

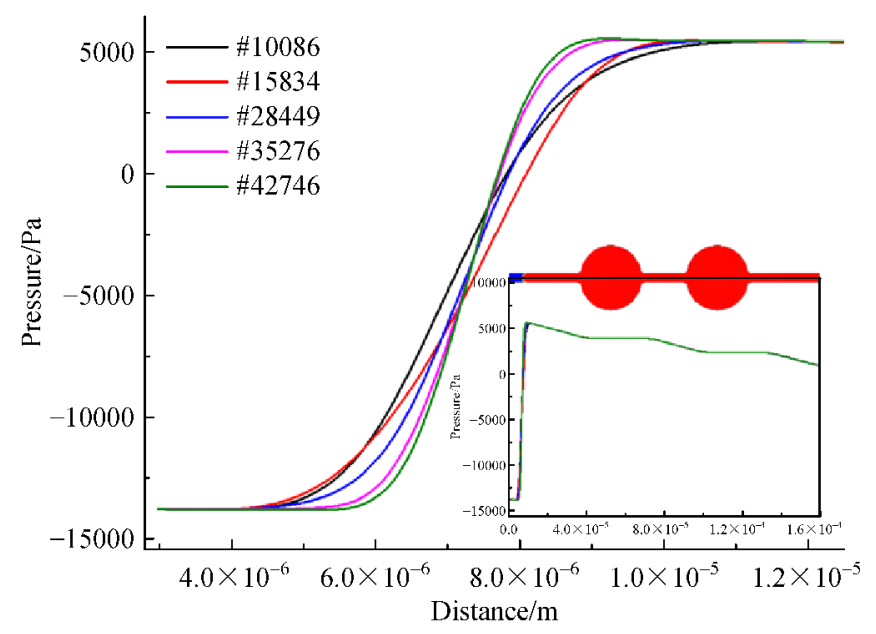

(a) selected for all simulations. From the comparison, it could be seen that the capillary pressure from the simulation satisfies the Laplace theory quite well.

\subsection{Snap-off phenomenon of oil phase in water-wet pores}

In this section, water invasion at the pore scale is modelled as the standard case. The water/oil phase distribution evolutions with time are shown in Fig. 4. It could be seen that the "snap-off" phenomenon can occur during the imbibition process, but only in the pore throat region. The phenomenon in which a wetting phase in the corners pinches off the non-wetting phase in the middle of the pore throat is referred to snap-off, which is a dominant mechanism for residual trapped oil in porous media. At the pore scale, capillary force plays an important role and can prevent the non-wetting phase from flowing. The snapoff process is mainly dependent on the physical geometry of pore channels, wettability and IFT [18]. At the first stage of the displacement, water tends to flow through the films, thus a swelling of the water film appears. As water invasion continues, the filling grows so that eventually the wetting fluid is able to fill the pore throat, making the snap-off happen. As the non-wetting phase cannot fill the next pore body rapidly and cannot keep the capillary pressure on the pore throat high enough, it is consequently pinched off by the wetting phase, which remains in the pore throat. This process continues intermittently until the second pore body is filled up, and the non-wetting phase in both pore bodies can stay connected without being snapped off. Ultimately, the oil phase is snapped off with seven discontinuous parts and stuck in the pore geometries, as shown in the final state in Fig. 4. This stuck phenomenon of the oil phase is consistent with previous experimental results [51], which demonstrated that a critical $\mathrm{Ca}$ to mobilize discontinuous oil is higher than that to mobilize continuous oil.

An example pressure distribution along the central axis

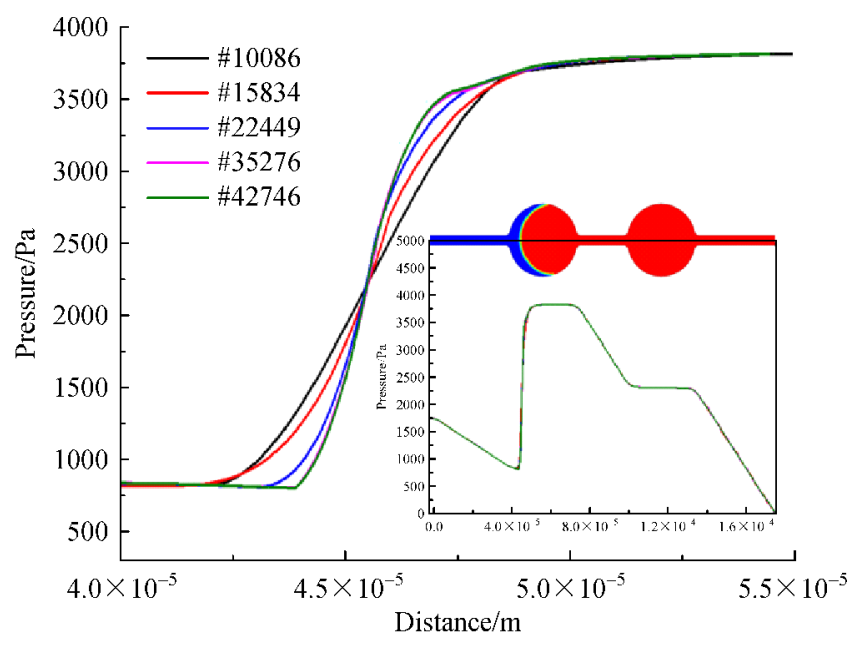

(b)

Fig. 3 Grid independency study for the initial oil-saturated channel case. (a) $t=10 \mathrm{~ms}$; (b) $t=100 \mathrm{~ms}$. 


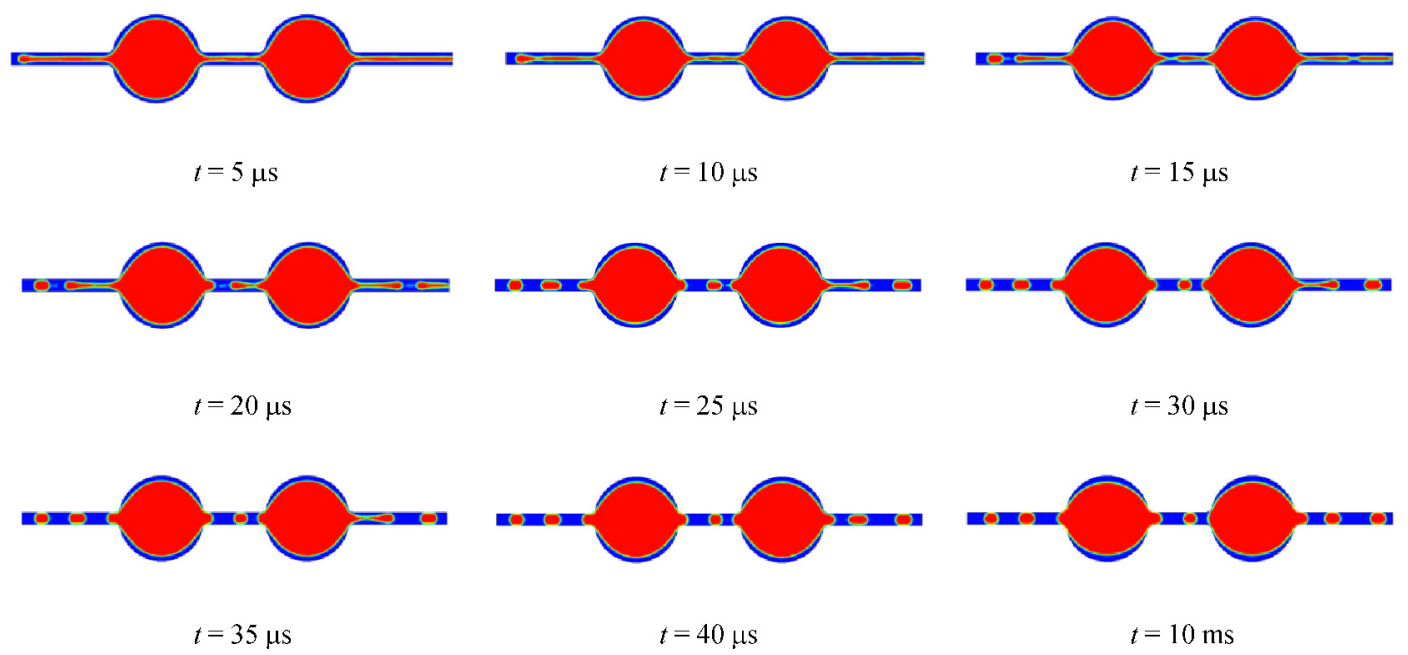

Fig. 4 Standard case: Traditional water flooding process, $C a=3.56 \times 10^{-5}$.

along horizontal direction after $1.0 \mathrm{~ms}$ is shown in Fig. 5 . Under the same condition, the smaller capillary diameter leads to a larger capillary pressure. Capillary force by a snapoff mechanism makes the oil phase unconnected, forming high pressure islands that prevent the recovery of oil.

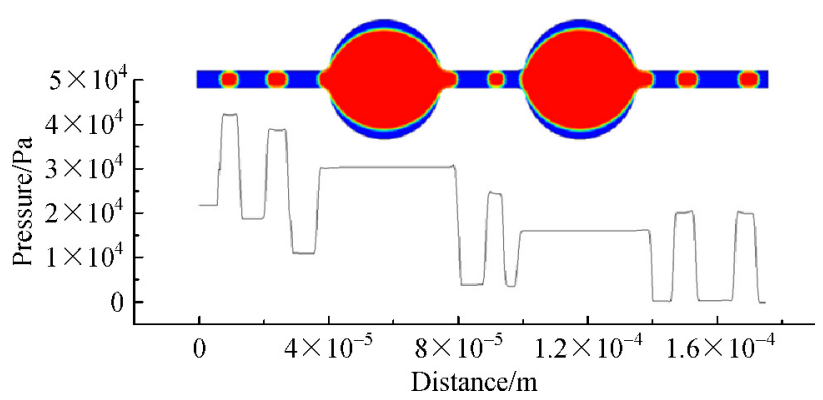

Fig. 5 The pressure distribution along the central axis of horizontal direction at the time evolutions of $1.0 \mathrm{~ms}$.

After the non-wetting phase forms pockets that are disconnected from the continuous phase, it is considered to be trapped and plug water channels. At the displacing time of $1.0 \mathrm{~ms}$, the remaining oil fraction in the pore channel is $73.97 \%$, which suggest that for this particular case simulated, traditional water flooding would not increase oil recovery rate significantly: nearly all the residual oil is trapped in the pore channel due to the capillary effect. In order to increase the $\mathrm{Ca}$ and suppress the snap-off phenomenon at the pore scale, the viscosity and IFT effects on the displacement for the water-wet pores are studied in the next section.

4.3 The effects of IFT and viscosity on the displacement for water-wet pores

The effects of IFT and viscosity on the displacement were simulated by 17 case studies, as shown in Table 1 . The simulation results of Case 1, as the standard case of traditional water flooding process, have been presented in the section 4.2. For the IFT effect study, the IFT between the invading fluid and oil phase was varied from 0.05 to $0.0005 \mathrm{~N} \cdot \mathrm{m}^{-1}$ at a given viscosity of $0.001 \mathrm{~Pa} \cdot \mathrm{s}$ (Cases $1-$ 9 ). To simulate the effect of the invading fluid viscosity, the viscosity of the invading fluid was varied from 0.001 to $0.05 \mathrm{~Pa} \cdot \mathrm{s}$ at a given IFT value of $0.05 \mathrm{~N} \cdot \mathrm{m}^{-1}$ (Cases $1,10^{-}$ 16). The corresponding $\mathrm{Ca}$ distribution is within the range of $3.56 \times 10^{-5}$ to $3.56 \times 10^{-3}$. An optimal case (Case 17) was also investigated with the minimum test IFT of 0.0005 $\mathrm{N} \cdot \mathrm{m}^{-1}$ and the maximum test viscosity of $0.05 \mathrm{~Pa} \cdot \mathrm{s}$, with a corresponding $\mathrm{Ca}$ of $1.780 \times 10^{-1}$.

Compared with the standard case, the simulation results of IFT and viscosity effects from Case 2 to Case 16 show that the pattern of the interface-shape evolution does not change dramatically, the snap-off phenomenon still occurs for all the cases at larger capillary numbers. However, the snap-off time was postponed significantly as compared with the traditional water flooding scenario and the number of the snapped-off positions was decreased (i.e., less than seven parts). For comparisons, the snap-off time in this paper refers to the time of the third break-up position for the oil phase. A dimensionless snap-off time $\tau$ is defined as $\tau=\tau_{\mathrm{s}} / \tau_{0}$, where $\tau_{0}$ is the standard snap-off time for traditional water flooding (Case 1). By using the dimensionless time and the $C a$, the snap-off time vs. the channel $\mathrm{Ca}$ is shown in Fig. 6. It can be seen that a lower IFT has stronger effects on postponing the snap-off time, which is beneficial for keeping the oil phase continuously.

The effects of viscosity and IFT on the oil recovery factor at the snap-off time are shown in Fig. 7. It can be observed that: (i) due to the snap-off phenomenon still existing, the oil recovery rates for all the cases are still remaining at a low level, (ii) though a low IFT flooding can postpone the snap-off time, larger viscosity of the invading 
Table 1 Parameters of the study on viscosity and IFT effects

\begin{tabular}{|c|c|c|c|c|}
\hline Case No. & $\mu_{0} /(\mathrm{Pa} \cdot \mathrm{s})$ & $\sigma /\left(\mathrm{N} \cdot \mathrm{m}^{-1}\right)$ & $\mathrm{Ca}$ & Viscosity ratio $\left(\mu_{\mathrm{o}} / \mu_{\mathrm{i}}\right)$ \\
\hline$\overline{1}$ & & 0.05 & $3.560 \times 10^{-5}$ & \\
\hline 2 & & 0.04 & $4.450 \times 10^{-5}$ & \\
\hline 3 & & 0.03 & $5.933 \times 10^{-5}$ & \\
\hline 4 & & 0.02 & $8.900 \times 10^{-5}$ & \\
\hline 5 & 0.001 & 0.01 & $1.780 \times 10^{-4}$ & $10: 1$ \\
\hline 6 & & 0.005 & $3.560 \times 10^{-4}$ & \\
\hline 7 & & 0.0025 & $7.120 \times 10^{-4}$ & \\
\hline 8 & & 0.001 & $1.780 \times 10^{-3}$ & \\
\hline 9 & & 0.0005 & $3.560 \times 10^{-3}$ & \\
\hline 10 & & 0.0025 & $8.900 \times 10^{-5}$ & $4: 1$ \\
\hline 11 & & 0.005 & $1.780 \times 10^{-4}$ & $2: 1$ \\
\hline 12 & & 0.01 & $3.560 \times 10^{-4}$ & $1: 1$ \\
\hline 13 & 0.05 & 0.02 & $7.120 \times 10^{-4}$ & $1: 2$ \\
\hline 14 & & 0.03 & $1.068 \times 10^{-3}$ & $1: 3$ \\
\hline 15 & & 0.04 & $1.424 \times 10^{-3}$ & $1: 4$ \\
\hline 16 & & 0.05 & $1.780 \times 10^{-3}$ & $1: 5$ \\
\hline 17 & 0.0005 & 0.05 & $1.780 \times 10^{-1}$ & $1: 5$ \\
\hline
\end{tabular}

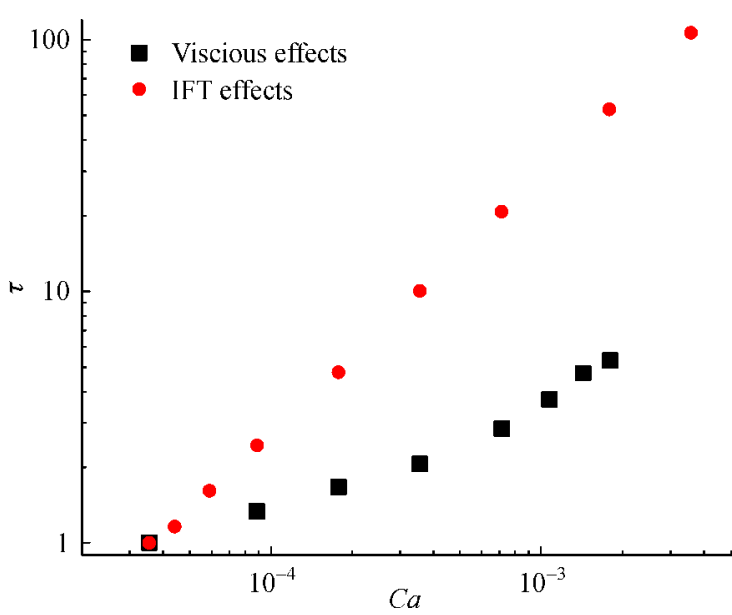

Fig. 6 The effects of viscosity and IFT on the snap-off dimensionless time.

fluid shows better recovery performance at the snap-off time. The viscosity effect on oil recovery is due to a decreased mobility difference between the displacing and displaced fluid by decreasing the capillary forces in comparison with viscous force, which is beneficial for oil recovery, (iii) different oil recovery rates can be obtained under the same $\mathrm{Ca}$ conditions, which differs from the macro-scale experimental conclusions, where it reported that the EOR effect is mainly dependent on the $\mathrm{Ca}$. A comparative example of water/oil mobilization profile by the IFT and viscosity effect respectively at a fixed $\mathrm{Ca}=$ $3.56 \times 10^{-4}$ is shown in Fig. 8 .

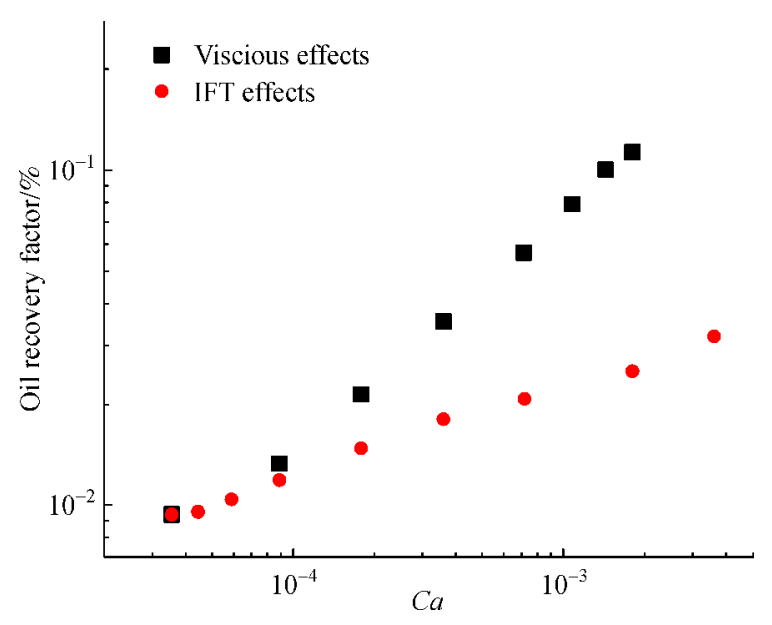

Fig. 7 Viscosity and IFT effects on oil recovery factor.

For the optimal case (Case 17) with the minimum test IFT of $0.0005 \mathrm{~N} \cdot \mathrm{m}^{-1}$ and the maximum test viscosity of $0.05 \mathrm{~Pa} \cdot \mathrm{s}$, which corresponding to a $\mathrm{Ca}$ of $1.780 \times 10^{-1}$, the water/oil phase distribution during the displacement process is shown in Fig. 9. It can be seen that although the collar in pore throats is growing in time, no snap-off is observed. A stable displacement process is achieved during the flooding, instead of snap-off or viscous fingering flow happening previously. This stable displacement contributes to nearly one hundred percentage of oil recovery, which represents the ideal situation for macroscale oil recovery. It could be concluded that by increasing the displacing viscosity and decreasing the IFT between oil 


The viscosity effect of $0.01 \mathrm{~Pa} \cdot \mathrm{s}$ at $C a=3.56 \times 10^{-4}$

Fig. 8 Comparisons of water/oil mobilization profile under the same $C a\left(C a=3.56 \times 10^{-4}\right)$ with the viscosity and IFT effect respectively.
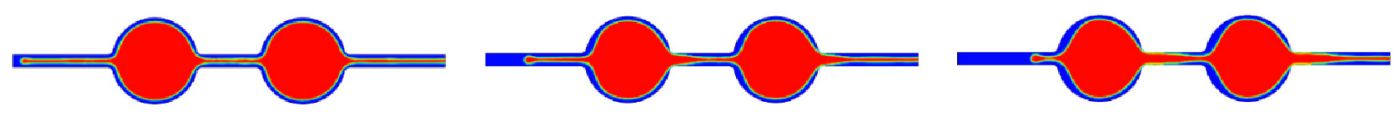

$t=2 \mathrm{~ms}$
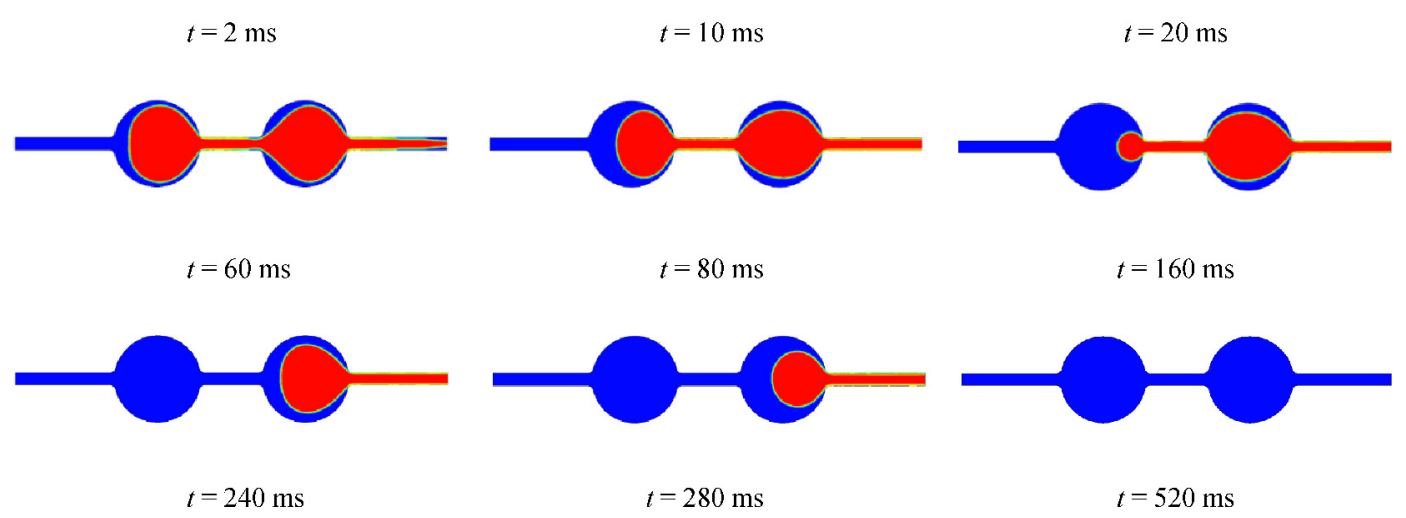

Fig. 9 Water/oil phase distribution during the displacement process for case $17\left(\mathrm{Ca}=1.780 \times 10^{-1}\right)$.

and water at the same time, a stable displacement could be achieved, instead of the snap-off phenomenon, which is beneficial to oil recovery in the field scale.

Figure 10 shows the pressure distribution profile along the centre of $x$ axis with the $C a$ of $1.780 \times 10^{-1}$ at the time of $2.0 \mu \mathrm{s}$. It can be observed that the pressure reduces gradually with the injection of invading fluid in the first stage, and it stays constant when water enters the middle pore. After water flows into the right throat, the pressure reduces continuously until the water breaks through. The pressure difference between the inlet and outlet is around $10000 \mathrm{~Pa}$, which is much smaller comparing to the traditional water flooding case, Fig. 5. In addition, the high-pressure islands associated with the snapped off phenomenon is disappeared.
4.4 The effects of IFT on the displacement process at the same $C a$

Traditional flooding theory claimed that capillary effects will have obviously negative influences on oil recovery in macroscale when $C a<10^{-5}$, due to the trapped effects. Above simulation suggests that the same $C a$ could render different oil recovery factors (RF) at the single pore scale. Therefore, this section will focus on the flow characteristic of flooding process for a given $\mathrm{Ca}$. With the same injecting velocity, four $\mathrm{Ca}$ number conditions with totally 28 scenarios were simulated here (i.e., in combination with different IFT and invading viscosities), which was $8.90 \times 10^{-5}, 3.56 \times 10^{-4}, 1.78 \times 10^{-3}$ and $1.78 \times 10^{-2}$, respectively. 


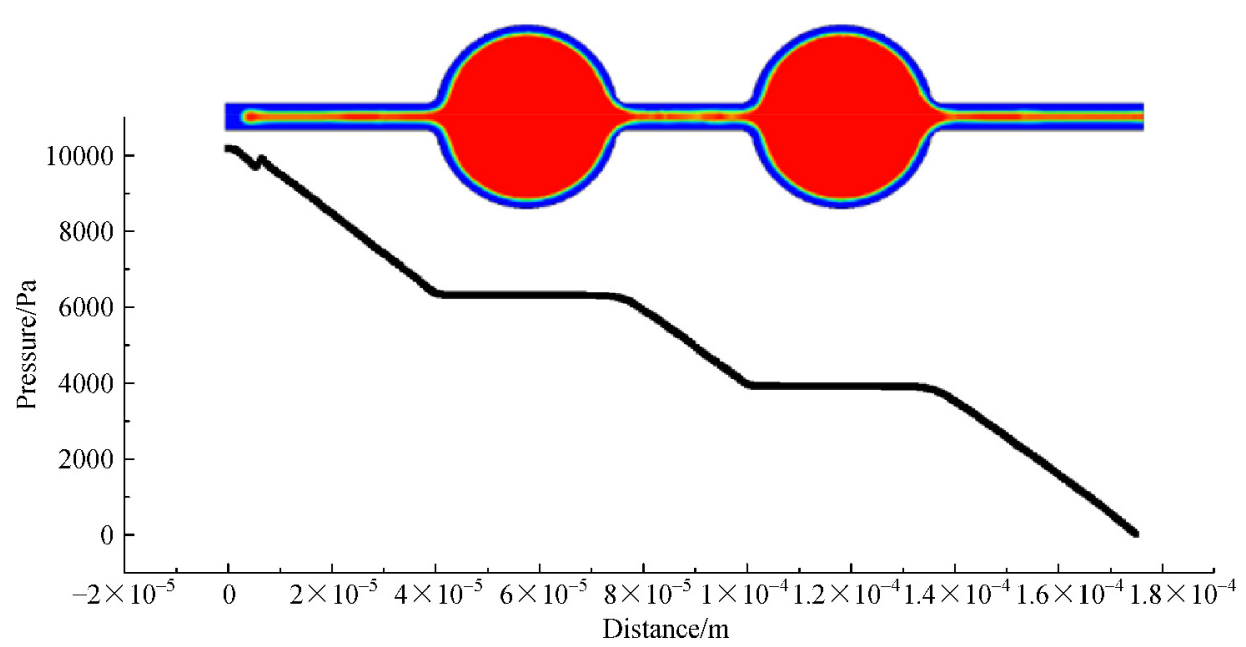

Fig. 10 The pressure distribution profile along the centre of $x$ axis for $C a=1.780 \times 10^{-1}$ at the time of $2.0 \mu \mathrm{s}$.

Three kinds of flow regimes are observed from the simulation results due to the effects of IFT and viscosity, which are snapped-off flow regime, transient flow regime and the stable flow regime, respectively. Hereinto, the snapped-off flow regime is defined as the final oil phase distribution with six or seven discontinuous parts; the stable flow regime refers to the displacement process with no snap-off position appearance, though with a tendency towards snapped-off due to the capillary effect; the flow with one to five discontinuous oil phase is termed as the transient flow regimes, which lies between the snapped-off and the stable displacement state. An example of each flow regime is shown in Fig. 11.

The flow regime distributions of all the 28 cases and the corresponding final oil recovery rates are concluded in Fig. 12. The scatters of the IFT, viscosity and the final EOR recovery factor are plotted on a logarithmic scale. Three colours of each scatter are corresponding with the three flow regime types, which are blue, dark yellow and magenta respectively. The red colour is used to represent the pore-scale EOR recovery factor for these 28 scenarios, which is shown in the right $y$ axis. The displacement scenarios with same capillary numbers are indicated by four shapes of points. The slopes of the four orange dash lines are all the same, which can reflect that the displacements are conducted with the same injecting and average velocity conditions. Additional 17 cases mentioned in Sect. 4.3 are also plotted with cross mark as the supplementary comparison. For the pore structure studied in this paper with the completely water-wet state, the simulation results show that: (i) by altering the invading viscosity and IFT between two phases, oil different RF and flow characteristics during the displacement process can be obtained, (ii) the same $\mathrm{Ca}$ can result in different oil RF due to the existence of different flow regimes, (iii) the critical $\mathrm{Ca}$ for mobilizing all the oil phase in this strongly waterwet pores is at a level of between $10^{-3}$ and $10^{-2}$, which is consistent with previous experimental results [52], (iv) different flow regimes are related to different pore-scale EOR RF: the snapped-off regime mainly leads to a RF around zero percentage; the transient flow can result in $2 \%-5 \%$ of oil recovery; and nearly one hundred percentage for the stable displacement process, (v) the three flow regime distributions can be divided into three separate zones, separated by the two black dash lines in this figure, which is more related to the variations of IFT than the invading viscosity.

4.5 The effect of the ultralow IFT on the displacement process

Above studies have showed that reducing IFT of oil-water is one of the most important mechanisms for enhancing oil recovery. However, a common belief that ultralow IFT (i.e., less than the order of magnitude of $10^{-3} \mathrm{mN} \cdot \mathrm{m}^{-1}$ ) values are needed for good performance in EOR has been

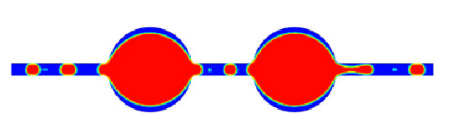

(a) Snapped-off flow

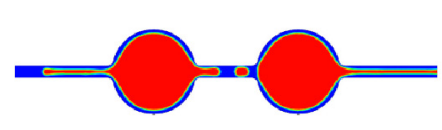

(b) Transient flow

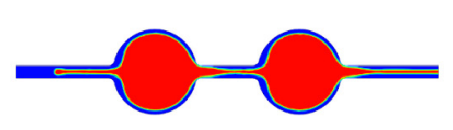

(c) Stable flow

Fig. 11 An example of the three flow regimes: (a) Snapped-off flow with $C a=1.78 \times 10^{-3}$, (b) Transient flow with $C a=1.78 \times 10^{-3}$, (c) stable flow regime with $\mathrm{Ca}=1.78 \times 10^{-2}$. 


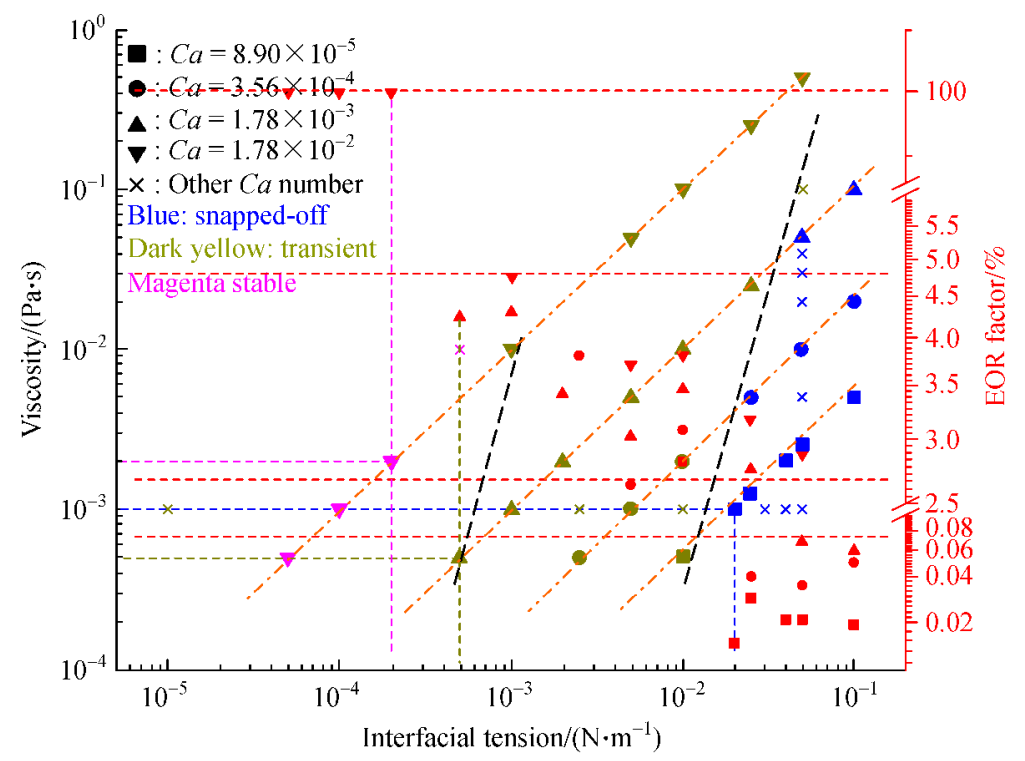

Fig. 12 Flow regime distributions of each case and the corresponding final EOR RF.

recently questioned [53]. To investigate if ultralow IFT is feasible for EOR, a case of IFT $=50 \mathrm{nN} \cdot \mathrm{m}^{-1}$ was simulated with a corresponding $\mathrm{Ca}$ of 35.6.

In a completely water-wet pore channel, the water/oil flow characterization results can be observed in Fig. 13. At the beginning of displacement, a stable interface advances as a concave meniscus. Then the interface turns to a convex meniscus and finally, it displays as a viscous fingering profile. Though there is no snap-off phenomenon happening in the capillary, there are still large quantities of oil remaining in the pore channel due to the fingering flow. The fingering has all the characteristics of the SaffmanTaylor [50] instability, which appears when a less viscous fluid pushes a more viscous fluid in a thin channel. The interface between the fluids develops an instability, leading to the formation of finger-like patterns. Similar fingering flow regime has also been observed experimentally by Zhang et al. [51] at an IFT $=50 \mathrm{nN} \cdot \mathrm{m}^{-1}$.

According to the numerical results, the remaining oil faction is around 0.32 at the breakthrough time of the displacing fluid, which leads to a pore-scale recovery factor of $56.80 \%$. Compared to traditional water flooding with the snap-off flow regime, the oil recovery factor is largely enhanced. However, nearly half of the oil is still remained in the pore channel. Such results support the recent questioning on the effect of IFT, i.e., an ultralow IFT may not achieve the expected EOR effect at a similar $\mathrm{Ca}$ [52]. Some recent flooding experiments [36] also showed that in some cases, there is no straightforward correlation between lowered IFT and enhanced recovery rate.

Figure 14 shows the pressure distribution along the centre of $x$ axis at $t=10 \mathrm{~ms}$. It can be seen that the pressure dropped slowly along the pore-scale channel during the flooding process. The pressure difference between the inlet and outlet is around $354.2 \mathrm{~Pa}$, which is much smaller than traditional water flooding and polymer/surfactant flooding. Therefore, chemical flooding with ultralow IFT could have encouraging effects of reducing injection pressure.

\section{Conclusions}

The effects of viscosity, IFT and $C a$ on the water/oil flow characteristics during the displacement process were simulated at the single pore scale by the VOF method. A pore-throat connecting channel was established to investigate the flooding process under water-wet condition. A flow regime was established revealing the different influences of viscosity and IFT. The simulation results can be concluded as the following:

(1) The initial continuous oil phase would be snapped off into several discontinuous oil ganglia by water and trapped into the pore channel due to the dominance of capillary effect, which would cause a low oil recovery rate at the macro scale.

(2) For water-wet pores, the snap-off phenomenon can be weakened by increasing the invading viscosity or decreasing the IFT respectively. Decreasing IFT mainly keeps oil phase staying continuously, while the increasing viscosity of invading fluid could enhance the confined fluid mobilization. Both are beneficial to EOR at the pore scale.

(3) Through increasing the invading viscosity and decreasing the IFT at the same time, the oil recovery factor would be efficiently enhanced by altering the flow regime from the snapped-off regime to the stable displacement condition.

(4) For water-wet pores, the same $\mathrm{Ca}$ can lead to different flow regimes and oil RF, due to the different 

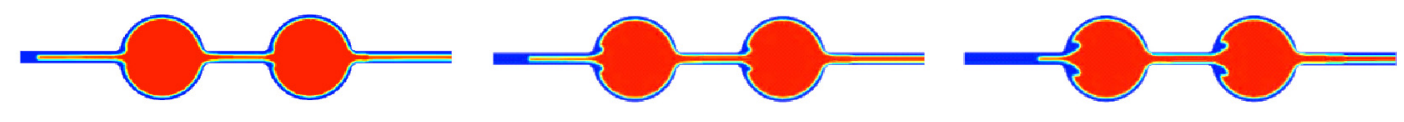

$t=5 \mathrm{~ms}$

$t=10 \mathrm{~ms}$

$t=20 \mathrm{~ms}$
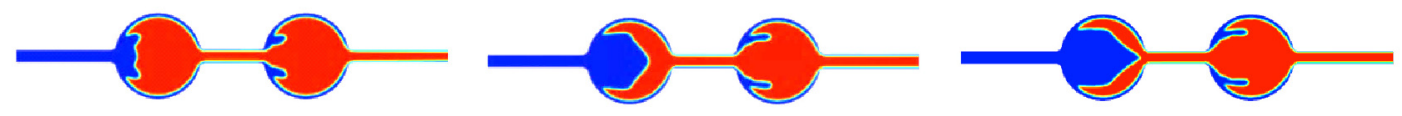

$t=40 \mathrm{~ms}$

$t=60 \mathrm{~ms}$

$t=100 \mathrm{~ms}$
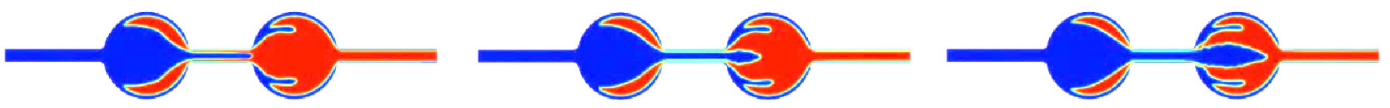

$t=120 \mathrm{~ms}$

$t=160 \mathrm{~ms}$

$t=180 \mathrm{~ms}$
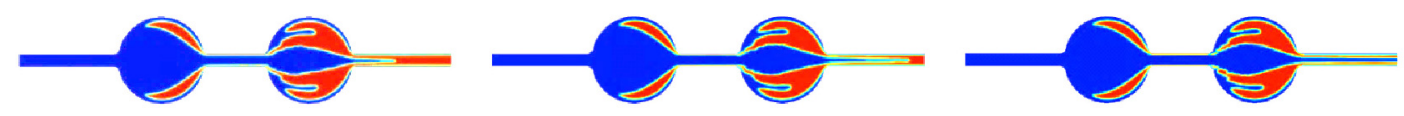

$t=200 \mathrm{~ms}$

$t=220 \mathrm{~ms}$

$t=240 \mathrm{~ms}$

Fig. 13 Viscous fingering at ultralow IFT $\left(\sigma=50 \mathrm{nN} \cdot \mathrm{m}^{-1}\right)$.

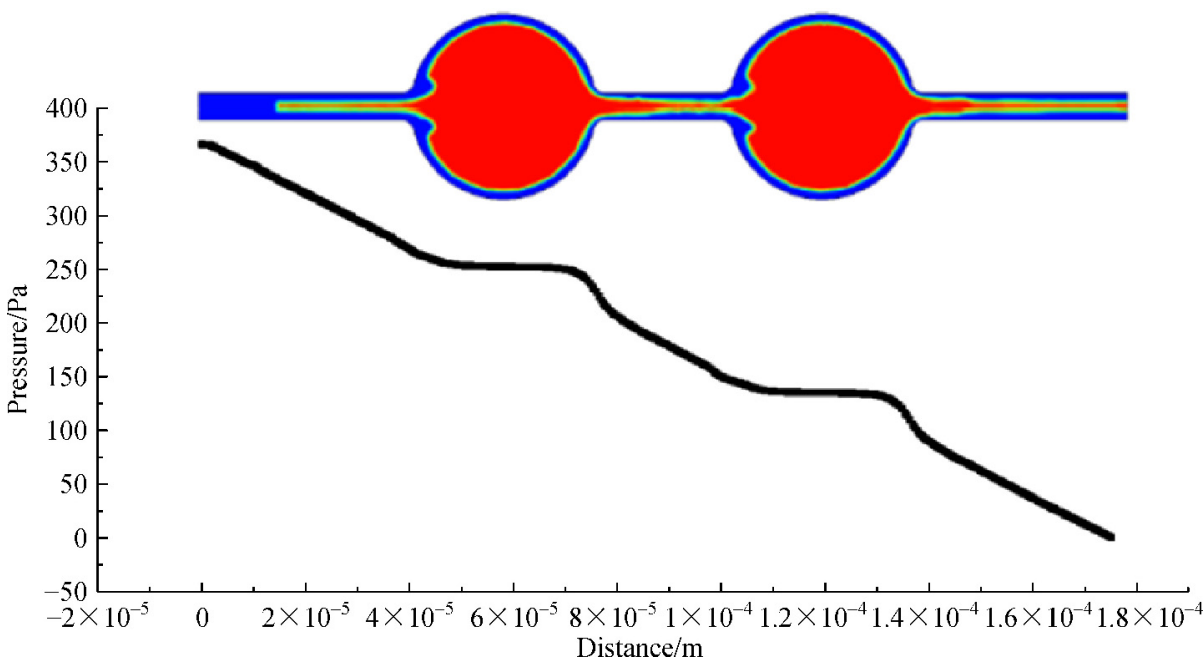

Fig. 14 The pressure distribution profile along the centre of $x$ axis at ultralow IFT $\left(\sigma=50 \mathrm{nN} \cdot \mathrm{m}^{-1}\right)$ at the time evolution of $10 \mathrm{~ms}$.

effects from viscosity and IFT. For the pore geometry studied in this paper, the snapped-off regime mainly can lead to a very small recovery factor; the transient flow can result in $2 \%-5 \%$ of oil recovery, and a one hundred percentage of recovery factor can be obtained with the stable flow at the single pore scale.

(5) When the IFT is decreased to an ultralow level, viscous fingering regime would happen in the pore channel though the injecting pressure can be reduced. A large $\mathrm{Ca}$ would not always lead to a large recovery factor.

(6) A stable displacement process can be achieved by properly increasing the displacing viscosity and decreasing the IFT between oil and water. Ultralow oil/water IFT is not necessarily due to the fingering possibility.

Acknowledgements This work was supported by European Research Council Consolidator Grant (Grant No. 648375) and the China Scholarship Council.

Open Access This article is licensed under a Creative Commons Attribution 4.0 International License, which permits use, sharing, adaptation, distribution and reproduction in any medium or format, as long as you give appropriate credit to the original author(s) and the source, provide a link to the Creative Commons licence, and indicate if changes were made. The images or other third party material in this article are included in the article's Creative Commons licence, unless indicated otherwise in a credit line to the material. 
If material is not included in the article's Creative Commons licence and your intended use is not permitted by statutory regulation or exceeds the permitted use, you will need to obtain permission directly from the copyright holder. To view a copy of this licence, visit http://creativecommons.org/licenses/by/4.0/.

\section{References}

1. Setiawan A, Suekane T, Deguchi Y, Kusano K. Three-dimensional imaging of pore-scale water flooding phenomena in water-wet and oil-wet porous media. Journal of Flow Control. Measurement \& Visualization, 2014, 2(2): 25-31

2. Schlüter S, Berg S, Rücker M, Armstrong R T, Vogel H J, Hilfer R, Wildenschild D. Pore-scale displacement mechanisms as a source of hysteresis for two-phase flow in porous media. Water Resources Research, 2016, 52(3): 2194-2205

3. Kallel W, van Dijke M I J, Sorbie K S, Wood R, Jiang Z, Harland S. Modelling the effect of wettability distributions on oil recovery from microporous carbonate reservoirs. Advances in Water Resources, 2016, 95: 317-328

4. Raeini A Q, Bijeljic B, Blunt M J. Modelling capillary trapping using finite-volume simulation of two-phase flow directly on microCT images. Advances in Water Resources, 2015, 83: 102-110

5. Andrew M, Bijeljic B, Blunt M J. Pore-scale contact angle measurements at reservoir conditions using X-ray microtomography. Advances in Water Resources, 2014, 68: 24-31

6. Arns C H, Knackstedt M A, Pinczewski W V, Garboczi E J. Computation of linear elastic properties from microtomographic images: Methodology and agreement between theory and experiment. Geophysics, 2002, 67(5): 1396-1405

7. Meakin P, Tartakovsky A M. Modeling and simulation of pore-scale multiphase fluid flow and reactive transport in fractured and porous media. Reviews of Geophysics, 2009, 47(3): 1-47

8. Tryggvason G, Bunner B, Esmaeeli A, Juric D, Al-Rawahi N, Tauber W, Han J, Nas S, Jan Y J. A front-tracking method for the computations of multiphase flow. Journal of Computational Physics, 2001, 169(2): 708-759

9. Fukai J, Shiiba Y, Yamamoto T, Miyatake O, Poulikakos D, Megaridis C M, Zhao Z. Wetting effects on the spreading of a liquid droplet colliding with a flat surface: Experiment and modeling. Physics of Fluids, 1995, 7(2): 236-247

10. Hirt C W, Nichols B D. Volume of fluid (VOF) method for the dynamics of free boundaries. Journal of Computational Physics, 1981, 39(1): 201-225

11. Lv M, Wang S. Pore-scale modeling of a water/oil two-phase flow in hot water flooding for enhanced oil recovery. RSC Advances, 2015, 5(104): 85373-85382

12. Ferrari A, Lunati I. Direct numerical simulations of interface dynamics to link capillary pressure and total surface energy. Advances in Water Resources, 2013, 57: 19-31

13. Brackbill J U, Kothe D B, Zemach C. A continuum method for modeling surface tension. Journal of Computational Physics, 1992, 100(2): 335-354

14. Afkhami S, Zaleski S, Bussmann M. A mesh-dependent model for applying dynamic contact angles to VOF simulations. Journal of Computational Physics, 2009, 228(15): 5370-5389
15. Tomin P, Lunati I. Hybrid multiscale finite volume method for twophase flow in porous media. Journal of Computational Physics, 2013, 250: 293-307

16. Yang X, Scheibe T D, Richmond M C, Perkins W A, Vogt S J, Codd S L, Seymour J D, McKinley M I. Direct numerical simulation of pore-scale flow in a bead pack: Comparison with magnetic resonance imaging observations. Advances in Water Resources, 2013, 54: 228-241

17. Horgue P, Augier F, Duru P, Prat M, Quintard M. Experimental and numerical study of two-phase flows in arrays of cylinders. Chemical Engineering Science, 2013, 102: 335-345

18. Roof J G. Snap-off of oil droplets in water-wet pores. Society of Petroleum Engineers Journal, 1970, 10(1): 85-90

19. Kiss É, Pintér J, Wolfram E. Dynamics of oil/water displacement in capillary tubes with periodically varying diameter. Colloid \& Polymer Science, 1982, 260(8): 808-810

20. Yu L, Wardlaw N C. The influence of wettability and critical porethroat size ratio on snap-off. Journal of Colloid and Interface Science, 1986, 109(2): 461-472

21. Ransohoff T C, Gauglitz P A, Radke C J. Snap-off of gas bubbles in smoothly constricted noncircular capillaries. AIChE Journal. American Institute of Chemical Engineers, 1987, 33(5): 753-765

22. Ransohoff T C, Radke C J. Laminar flow of a wetting liquid along the corners of a predominantly gas - occupied noncircular pore. Journal of Colloid and Interface Science, 1988, 121(2): 392401

23. Al-Gharbi M S, Blunt M J. Dynamic network modeling of twophase drainage in porous media. Physical Review. E, 2005, 71(1): 016308

24. Al-Gharbi M S. Dynamic pore-scale modelling of twophase flow. Dissertation for the Doctoral Degree. London: University of London, 2004, 1-132

25. Beresnev I A, Deng W. Theory of breakup of core fluids surrounded by a wetting annulus in sinusoidally constricted capillary channels. Physics of Fluids, 2010, 22(1): 012105

26. Beresnev I A, Li W, Vigil R D. Condition for break-up of nonwetting fluids in sinusoidally constricted capillary channels. Transport in Porous Media, 2009, 80(3): 581-604

27. Deng W. Quantitative aspects of vibratory mobilization and breakup of non-wetting fluids in porous media. Dissertation for the Doctoral Degree. Iowa: Iowa State University, 2010, 1-124

28. Deng W, Cardenas M B. Dynamics and dislodgment from pore constrictions of a trapped nonwetting droplet stimulated by seismic waves. Water Resources Research, 2013, 49(7): 4206-4218

29. Deng W, Cardenas M B, Bennett P C. Extended Roof snap-off for a continuous nonwetting fluid and an example case for supercritical $\mathrm{CO}_{2}$. Advances in Water Resources, 2014, 64: 34-46

30. Roman S, Abu-Al-Saud M O, Tokunaga T, Wan J, Kovscek A R, Tchelepi H A. Measurements and simulation of liquid films during drainage displacements and snap-off in constricted capillary tubes. Journal of Colloid and Interface Science, 2017, 507: 279-289

31. Abu-Al-Saud M O, Riaz A, Tchelepi H A. Multiscale level-set method for accurate modeling of immiscible two-phase flow with deposited thin films on solid surfaces. Journal of Computational Physics, 2017, 333: 297-320

32. Deng W, Balhoff M, Cardenas M B. Influence of dynamic factors on 
nonwetting fluid snap-off in pores. Water Resources Research, 2015, 51(11): 9182-9189

33. Raffa P, Broekhuis A A, Picchioni F. Polymeric surfactants for enhanced oil recovery: A review. Journal of Petroleum Science Engineering, 2016, 145: 723-733

34. Hirasaki G, Miller C A, Puerto M. Recent advances in surfactant EOR. SPE Journal, 2011, 16(04): 889-907

35. Ko K M, Chon B H, Jang S B, Jang H Y. Surfactant flooding characteristics of dodecyl alkyl sulfate for enhanced oil recovery. Journal of Industrial and Engineering Chemistry, 2014, 20(1): 228-233

36. Yu Q, Jiang H, Zhao C. Study of interfacial tension between oil and surfactant polymer flooding. Petroleum Science and Technology, 2010, 28(18): 1846-1854

37. Samanta A, Ojha K, Sarkar A, Mandal A. Surfactant and surfactantpolymer flooding for enhanced oil recovery. Advances in Petroleum Exploration and Development, 2011, 2(1): 13-18

38. Olajire A A. Review of ASP EOR (alkaline surfactant polymer enhanced oil recovery) technology in the petroleum industry: Prospects and challenges. Energy, 2014, 77: 963-982

39. Raffa P, Wever D A Z, Picchioni F, Broekhuis A A. Polymeric surfactants: Synthesis, properties, and links to applications. Chemical Reviews, 2015, 115(16): 8504-8563

40. Liu R C, Pallier A, Brestaz M, Pantoustier N, Tribet C. Impact of polymer microstructure on the self-assembly of amphiphilic polymers in aqueous solutions. Macromolecules, 2007, 40(12): 4276-4286

41. Meybodi H E, Kharrat R, Wang X. Study of microscopic and macroscopic displacement behaviors of polymer solution in waterwet and oil-wet media. Transport in Porous Media, 2011, 89(1): 97120

42. Wever D A Z, Picchioni F, Broekhuis A A. Polymers for enhanced oil recovery: A paradigm for structure-property relationship in aqueous solution. Progress in Polymer Science, 2011, 36(11): 15581628

43. Abidin A Z, Puspasari T, Nugroho W A. Polymers for enhanced oil recovery technology. Procedia Chemistry, 2012, 4: 11-16

44. Brackbill J U, Kothe D B, Zemach C. A continuum method for modeling surface tension. Journal of Computational Physics, 1992, 100(2): 335-354

45. Bagrintseva K I. Carbonate Reservoir Rocks. New Jersey: Wiley, 2015, 1-285

46. Nelson P H. Pore-throat sizes in sandstones, tight sandstones, and shales. AAPG Bulletin, 2009, 93(3): 329-340

47. Dake L P. The Practice of Reservoir Engineering. Amsterdam: Elsevier, 2013, 1-518

48. White F M, Corfield I. Viscous Fluid Flow. New York: McGrawHill, 2006, 1-578

49. Bear J. Dynamics of Fluids in Porous Media. Massachusetts: Courier Corporation, 2013, 1-725

50. Setu S A, Zacharoudiou I, Davies G J, Bartolo D, Moulinet S, Louis A A, Yeomans J M, Aarts D G A L. Viscous fingering at ultralow interfacial tension. Soft Matter, 2013, 9(44): 10599-10605

51. Zhang H, Dong M, Zhao S. Which one is more important in chemical flooding for enhanced court heavy oil recovery, lowering interfacial tension or reducing water mobility? Energy \& Fuels, 2010, 24(3): 1829-1836

52. Saffman P G, Taylor G I. The penetration of a fluid into a porous medium or Hele-Shaw cell containing a more viscous liquid. Proceedings of the Royal Society of London, 1958, 245(1242): 312329

53. Sheng J J. Preferred calculation formula and buoyancy effect on capillary number. Asia-Pacific Journal of Chemical Engineering, 2015, 10(3): 400-410 